IUHET-444

September 2001

\title{
Leptogenesis and Low-energy Observables
}

\author{
M. S. Berger * and Kim Siyeon ${ }^{\dagger}$ \\ Physics Department, Indiana University, Bloomington, IN 47405, USA
}

\begin{abstract}
We relate leptogenesis in a class of theories to low-energy experimental observables: quark and lepton masses and mixings. With reasonable assumptions motivated by grand unification, one can show that the CP-asymmetry parameter takes a universal form. Furthermore the dilution mass is related to the light neutrino masses. Overall, these models offer an explanation for a lepton asymmetry in the early universe.
\end{abstract}

*Electronic address: berger@gluon.physics.indiana.edu

${ }^{\dagger}$ Electronic address: siyeon@gluon.physics.indiana.edu 


\section{INTRODUCTION}

Recent compelling evidence for neutrino oscillations has accelerated work on formulating theoretical models for fermion masses and mixings. The current data indicates that there are most likely two large mixing angles and one small one in the lepton sector. The first large mixing angle arises in the atmospheric neutrino data, while it is becoming increasingly likely that the solar neutrino data is described by an Mikheyev-Smirnov-Wolfenstein-(MSW) type oscillation with a large mixing angle (LMA) 표. 4 . On the other hand the CHOOZ experiment [5] gives an upper bound on the third mixing angle. A best fit [6] for the atmospheric neutrino data and the LMA solution for the solar neutrino data is

$$
\begin{gathered}
\Delta m_{32}^{2}=3.2 \times 10^{-3} \mathrm{eV}^{2}, \\
\sin ^{2} 2 \theta_{23}=1.000 \\
\Delta m_{21}^{2}=3.2 \times 10^{-5} \mathrm{eV}^{2}, \\
\sin ^{2} 2 \theta_{12}=0.75, \tan ^{2} \theta_{12}=0.33,
\end{gathered}
$$

The observations of neutrino mixing and the measured values for the differences in masssquareds make very plausible the existence of heavy Majorana neutrinos, $\nu_{M_{i}}$. These neutrinos can naturally be very heavy since they are Standard Model gauge singlets and their masses are not connected to the breaking of the electroweak symmetry. These heavy Majorana neutrinos existed in the early universe and can have $\mathrm{CP}$ violating decay modes. Therefore the heavy neutrinos are natural candidates for producing a lepton asymmetry via out-of-equilibrium decays. This asymmetry produced in the early universe is recycled into a baryon asymmetry by sphaleron transitions which violated both baryon number and lepton number. The resulting baryon asymmetry is the same order of magnitude as the original lepton asymmetry [7].

In the mass basis where the right-handed Majorana mass matrix $M_{R}$ is diagonal the asymmetry in heavy neutrino decays

$$
\epsilon_{i}=\frac{\Gamma\left(\nu_{M_{i}} \rightarrow \ell H_{2}\right)-\Gamma\left(\nu_{M_{i}} \rightarrow \ell^{c} H_{2}^{c}\right)}{\Gamma\left(\nu_{M_{i}} \rightarrow \ell H_{2}\right)+\Gamma\left(\nu_{M_{i}} \rightarrow \ell^{c} H_{2}^{c}\right)},
$$

is given by 813

$$
\epsilon_{i}=\frac{3}{16 \pi v_{2}^{2}} \frac{1}{\left(\mathcal{N}^{\dagger} \mathcal{N}\right)_{i i}} \sum_{n \neq i} \operatorname{Im}\left[\left(\mathcal{N}^{\dagger} \mathcal{N}\right)_{n i}^{2}\right] \frac{M_{i}}{M_{n}}
$$

where $\mathcal{N}$ is the neutrino Dirac mass matrix in a weak basis. The masses $M_{i}$ are the three eigenvalues of the heavy Majorana mass matrix and $v_{2}$ is the vacuum expectation value (VEV) of the Higgs boson giving Dirac masses to the neutrinos and up-type quarks. $M_{1}$ is the mass of the lightest of the three heavy Majorana neutrinos, and Eq. (6) is an approximate formula valid for $M_{n}>>M_{i}$. When this is the case, the lepton asymmetry is generated by the decays of the lightest Majorana neutrino, $\nu_{M_{1}}$.

The size of the lepton asymmetry generated by $\nu_{M_{1}}$ decays is also strongly dependent on the size of a mass parameter sometimes called the dilution mass defined as 


$$
\tilde{m}_{1}=\frac{\left(\mathcal{N}^{\dagger} \mathcal{N}\right)_{11}}{M_{1}} .
$$

This parameter controls (a) the decay width of the lightest right-handed Majorana neutrino $\nu_{M_{1}}$ since

$$
\Gamma_{\nu_{M_{1}}}=\frac{1}{8 \pi}\left(\mathcal{N}^{\dagger} \mathcal{N}\right)_{11} \frac{M_{1}}{v_{2}^{2}},
$$

as well as (b) the amount of dilution caused by lepton number violating scattering: the resulting lepton asymmetry depends critically on the parameter $\tilde{m}_{1}$ because it governs the size of the most important Yukawa coupling in the $\Delta L=2$ scattering processes, as has been shown in detail in numerical calculations [7,9, 11, 14, 150]. These two constraints bound the possible values of $\tilde{m}_{1}$ such that a sufficient asymmetry is produced to agree with observation. The generated lepton asymmetry $Y_{L}$ is defined in terms of the number densities of the leptons and antileptons as well as the entropy density as

$$
Y_{L}=\frac{n_{L}-n_{\bar{L}}}{s}=\kappa \frac{\epsilon_{1}}{g^{*}},
$$

where $g^{*}$ is the number of light (effective) degrees of freedom in the theory, and $\kappa$ is a dilution factor that can be reliably calculated by solving the full Boltzmann equations.

It has been shown [9, 11] that a CP-violation parameter $\epsilon_{1} \sim 10^{-6}$ and a dilution mass $\tilde{m}_{1}$ in the range of the light neutrino masses can produce the sufficient amount of leptogenesis to account for the observed baryon asymmetry. From the definition of the dilution mass in Eq. (]) it is clear that the dilution mass will indeed be related to the light neutrino masses in most models. It is a nontrivial occurrence that the amount of baryon asymmetry of the universe is obtained from a recycling of the leptogenesis that naturally occurs via Majorana neutrino decays.

Suppose one starts in a basis where $M_{R}$ is diagonal with eigenvalues $M_{j}$, and suppose the matrix $M_{R}$ is connected to the light neutrino mass matrix $m_{\nu}$ by a seesaw mechanism,

$$
M_{R}=\mathcal{N}^{T} m_{\nu}^{-1} \mathcal{N} .
$$

One can then define mixing matrices $U_{L, R}^{(N)}$ and $V_{L}$ that diagonalize $N$ and $m_{\nu}$ respectively,

$$
\begin{aligned}
& \mathcal{N}=U_{L}^{(N)} N_{\text {diag }} U_{R}^{(N) \dagger} \\
& \mathcal{N}^{T}=U_{R}^{(N) *} N_{\text {diag }} U_{L}^{(N) T} \\
& m_{\nu}^{-1}=V_{L}^{*} m_{\text {diag }}^{-1} V_{L}^{\dagger} .
\end{aligned}
$$

With these transformation matrices defined, $M_{j}$ can be written in terms of mass eigenvalues and mixings of $\left(m_{\nu}, \mathcal{N}\right)$,

$$
M_{j}=\sum_{k} \sum_{\ell} m_{k}^{-1}\left(V_{L}^{\dagger} U_{L}^{(N)}\right)_{k \ell}^{2} N_{\ell}^{2} U_{R \ell j}^{(N) \dagger 2}
$$

where $N_{l}$ are the diagonal elements of $N_{\text {diag }}$. The unitary transformation $U_{R}^{(N)}$ diagonalizes $\mathcal{N}^{\dagger} \mathcal{N}$ as $\mathcal{N}^{\dagger} \mathcal{N}=U_{R}^{(N)} N_{\text {diag }}^{2} U_{R}^{(N) \dagger}$, then 


$$
\left(\mathcal{N}^{\dagger} \mathcal{N}\right)_{1 j}=\sum_{k} N_{k}^{2} U_{R 1 k}^{(N)} U_{R j k}^{(N) *}
$$

By inverting $M_{j}$ in Eq. (14), the mass eigenvalues of $m_{\nu}$ can be expressed in terms of $V_{L}$, mixing angles and eigenvalues of Dirac mass matrix, and Majorana neutrino masses,

$$
m_{k}=\sum_{j} \sum_{\ell}\left(V_{L}^{\dagger} U_{L}^{(N)}\right)_{k \ell}^{2} N_{\ell}^{2} U_{R \ell j}^{(N) \dagger 2} M_{j}^{-1}
$$

\section{ASSUMPTIONS}

It is well-known that one must make theoretical assumptions about the structure of the neutrino masses and mixings to make progress in ascertaining whether leptogenesis is viable. For example the source of CP-violation responsible for producing the CP-violating decays of heavy Majorana neutrinos (and hence giving rise to leptogenesis) does not have to be related to the CP-violation that might be measurable at low-energy experiments in the future [16,17]. An extensive study of the weak-basis CP invariants in models with three iso-singlet neutrinos is given in Ref. [18]. If one makes the assumption of single righthanded neutrino dominance, then the low energy neutrino observables and the leptogenesis predictions decouple entirely [19]. On the other hand, in certain classes of grand unified theories previously unconstrained parameters become related to observables. For example, in models with a left-right symmetry, the right-handed mixing angles can be related the left-handed ones that enter into low-energy experiments [20]. In this section we list our theoretical assumptions about the underlying grand unified theory. Many authors have discussed leptogenesis in the context of grand unified theories [21 36]; our emphasis here is on making the most general assumptions that allow us to relate low-energy observables like masses and mixing angles to the required lepton asymmetry that can ultimately account for the baryon asymmetry of the universe.

[A1] We assume that the Dirac mass matrices $\mathcal{N}$ and $\mathcal{U}$ are symmetric, and $\mathcal{N} \sim \mathcal{U}$ ]. This similarity between the neutrino Dirac mass matrix and the up-quark mass matrix is motivated by grand unified theories.

[A2] The mixing angles contained in the transformation matrices that diagonalize the neutrino Dirac mass matrix $\mathcal{N}$ are related to the eigenvalues $1 s_{i j} \sim \sqrt{\frac{N_{i}}{N_{j}}}$. In general these mixing angles cannot be larger than $\sqrt{\frac{N_{i}}{N_{j}}}$, but can in principle be smaller. The $s_{i j}$ being suppressed compared to $\sqrt{\frac{N_{i}}{N_{j}}}$ might occur, for example, if some elements of $\mathcal{N}$ are suppressed or zero. So the result of our second assumption is that there is no such suppression or cancellation in the Dirac neutrino matrix.

\footnotetext{
${ }^{1}$ We use the notation $\sim$ to denote that entries are the same size to leading order in all small quantities such as small mass ratios or small mixing angles.

${ }^{2}$ We use the shorthand notation $c_{i j} \equiv \cos \theta_{i j}$ and $s_{i j} \equiv \sin \theta_{i j}$.
} 
The crucial features that follow from our two assumptions listed above are (a) the neutrino Dirac mass matrix has eigenvalues that mimic the large hierarchy that exists in the up-quark sector, and (b) the mixing angles $s_{i j}$ are fixed to be of some definite size related to the uptype quark masses, e.g. $s_{13} \sim \sqrt{N_{1} / N_{3}} \sim \sqrt{m_{u} / m_{t}}$. These two results will be important in arriving at the relatively simple results that follow.

[A3] Our approach does not allow us to determine the CP-violating phase that enters into the parameter $\epsilon_{1}$ in Eq. (6). We simply assume that phases are of order one, and there is no suppression arising from unnaturally small parameters.

A standard parametrization of the unitary transformation involving three angles and a phase is

$$
\begin{aligned}
U & =\left(\begin{array}{ccc}
1 & 0 & 0 \\
0 & c_{23} & s_{23} \\
0 & -s_{23} & c_{23}
\end{array}\right)\left(\begin{array}{ccc}
c_{13} & 0 & s_{13} e^{-i \delta} \\
0 & 1 & 0 \\
-s_{13} e^{i \delta} & 0 & c_{13}
\end{array}\right)\left(\begin{array}{ccc}
c_{12} & s_{12} & 0 \\
-s_{12} & c_{12} & 0 \\
0 & 0 & 1
\end{array}\right) \\
& =\left(\begin{array}{ccc}
c_{13} c_{12} & s_{12} c_{13} & s_{13} e^{-i \delta} \\
-s_{12} c_{23}-s_{23} s_{13} c_{12} e^{i \delta} & c_{23} c_{12}-s_{23} s_{13} s_{12} e^{i \delta} & s_{23} c_{13} \\
s_{23} s_{12}-s_{13} c_{23} c_{12} e^{i \delta} & -s_{23} c_{12}-s_{13} s_{12} c_{23} & c_{23} c_{13}
\end{array}\right) .
\end{aligned}
$$

The right-handed and left-handed mixing matrices with small angles $\left(c_{i j} \approx 1\right)$ are

$$
U_{R}^{(N)} \approx U_{L}^{(N)} \approx\left(\begin{array}{ccc}
1 & s_{12} & s_{13} \\
-s_{12}-s_{23} s_{13} & 1 & s_{23} \\
s_{23} s_{12}-s_{13} & -s_{23}-s_{13} s_{12} & 1
\end{array}\right)
$$

where we will assume that phase $e^{i \delta}$ is not suppressed: $\delta$ is not close to 0 or $\pi$. For our purposes, we consider only the leading contributions to each element so that

$$
\left|U_{R}^{(N)}\right| \sim\left|U_{L}^{(N)}\right| \sim\left(\begin{array}{ccc}
1 & s_{12} & s_{13} \\
s_{12} & 1 & s_{23} \\
s_{13} & s_{23} & 1
\end{array}\right)
$$

\section{NEUTRINO TRANSFORMATION}

In general, we can write the transformation as

$$
V_{L} \sim\left(\begin{array}{ccc}
1 & \Theta_{12} & \Theta_{13} \\
-\Theta_{12}-\Theta_{23} \Theta_{13} & 1 & \Theta_{23} \\
\Theta_{23} \Theta_{12}-\Theta_{13} & -\Theta_{23}-\Theta_{13} \Theta_{12} & 1
\end{array}\right)
$$

We henceforth interpret the quantities $\Theta_{i j}$ as

$$
\begin{array}{ll}
\cos \Theta \sim 1, & \sin \Theta \sim 1, \quad \text { for large angles } \\
\cos \Theta \sim 1, & \sin \Theta \sim \Theta, \quad \text { for small angles. }
\end{array}
$$

In other words, the matrix can be expressed in the same way in terms of $\Theta_{i j}$ if we are only interested in the order-of-magnitude size of the elements (including the ones on the diagonal 
which would only be of order one in general). The Maki-Nakagawa-Sakata (MNS) neutrino mixing matrix [37] is

$$
U_{M N S}=U_{L}^{(E) \dagger} V_{L}
$$

where $U_{L}^{(E)}$ is the matrix that diagonalizes the charged lepton mass matrix. The constraints from reactor neutrino mixing data [5] imply that $\Theta_{13}$ must be small provided there is no cancellation among $V_{L}$ and $U_{L}^{(E)}$. Retaining only information about the size of the individual elements, we may write Eq. (20) as follows

$$
V_{L} \sim\left(\begin{array}{ccc}
1 & \Theta_{12} & \Theta_{13} \\
\max \left(\Theta_{12}, \Theta_{23} \Theta_{13}\right) & 1 & \Theta_{23} \\
\max \left(\Theta_{23} \Theta_{12}, \Theta_{13}\right) & \Theta_{23} & 1
\end{array}\right),
$$

with the entries interpreted according to Eq. (21).

\section{HEAVY MAJORANA NEUTRINO MASSES}

Define the matrix

$$
W_{k j} \equiv \sum_{\ell}\left(V_{L}^{\dagger} U_{L}^{(N)}\right)_{k \ell}^{2} n_{\ell}^{2} U_{R \ell j}^{(N) \dagger 2}
$$

where $n_{i} \equiv N_{i} / N_{3}$ are the ratios of the Dirac neutrino masses. The heavy Majorana neutrino masses are

$$
M_{j}=N_{3}^{2} \sum_{k} m_{k}^{-1} W_{k j}
$$

and the light neutrino masses are given by Eq. (16) as

$$
m_{j}=N_{3}^{2} \sum_{k} W_{j k} M_{k}^{-1}
$$

The factor $n_{\ell}^{2} U_{R \ell j}^{(N) \dagger 2}$ in $W_{k j}$ has the form

$$
n_{\ell}^{2} U_{R \ell j}^{(N) \dagger 2} \sim\left(\begin{array}{ccc}
n_{1}^{2} & n_{1}^{2} s_{12}^{2} & n_{1}^{2} s_{13}^{2} \\
n_{2}^{2} s_{12}^{2} & n_{2}^{2} & n_{2}^{2} s_{23}^{2} \\
s_{13}^{2} & s_{23}^{2} & 1
\end{array}\right) .
$$

Now we make use of our assumptions [A1] and [A2] that allow us to compare the relative sizes of the $n_{i}$ and the mixing angles $s_{i j}$. Specifically we have that $s_{i j} \sim \sqrt{n_{i} / n_{j}}$ as well as $n_{i}<<n_{j}$ for $i<j$ so that

$$
n_{1}^{2} U_{R 1 j}^{(N) \dagger 2}<<n_{2}^{2} U_{R 2 j}^{(N) \dagger 2}<<U_{R 3 j}^{(N) \dagger 2} .
$$

We henceforth refer to this condition as "third-generation dominance." In fact if, as we have assumed, the hierarchy in the Dirac masses for neutrinos is as strong as it is for the up quark 
Dirac masses, as one might expect in a grand unified theory, then the smallness of $n_{1}$ and $n_{2}$ suppresses all other contributions to $W_{k j}$ relative to the dominant contribution coming from $\left(V_{L}^{\dagger} U_{L}^{(N)}\right)_{k 3}^{2}$ and $U_{R 3 j}^{(N) \dagger 2}$. So we arrive at the following factored form for the matrix

$$
W_{k j} \sim\left(\begin{array}{c}
\left(V_{L}^{\dagger} U_{L}^{(N)}\right)_{13}^{2} \\
\left(V_{L}^{\dagger} U_{L}^{(N)}\right)_{23}^{2} \\
\left(V_{L}^{\dagger} U_{L}^{(N)}\right)_{33}^{2}
\end{array}\right)\left(\begin{array}{lll}
s_{13}^{2} & s_{23}^{2} & 1
\end{array}\right) .
$$

Finally we can write the Majorana masses in the following way

$$
\left(M_{1}, M_{2}, M_{3}\right) \sim N_{3}^{2} \tilde{W}_{3}\left(s_{13}^{2}, s_{23}^{2}, 1\right)
$$

where

$$
\tilde{W}_{3}=\sum_{k} m_{k}^{-1}\left(V_{L}^{\dagger} U_{L}^{(N)}\right)_{k 3}^{2}
$$

The result in Eq. (30) indicates that, based on our assumptions, the mass ratios of the Majorana masses are related to the mixing angles $s_{i 3}$ and are independent of the light neutrino mixings which appear only in the overall factor $\tilde{W}_{3}$. This result follows from the third-generation dominance Eq. (28) which is related to the large hierarchy in the Dirac neutrino masses that is inherited from the large hierarchy in the experimentally measured up-quark masses. On the other hand, the light neutrino masses under the third-generation condition are given by Eq. (26) as

$$
\left(m_{1}, m_{2}, m_{3}\right) \sim \frac{N_{3}^{2}}{M_{3}}\left(\left(V_{L}^{\dagger} U_{L}^{(N)}\right)_{13}^{2},\left(V_{L}^{\dagger} U_{L}^{(N)}\right)_{23}^{2},\left(V_{L}^{\dagger} U_{L}^{(N)}\right)_{33}^{2}\right)
$$

So the mass ratios of the light neutrinos can be expressed in terms of the left-handed mixing angles.

\section{LEPTOGENESIS}

In this section we utilize the simple form for the mass ratios of the heavy Majorana neutrino masses found in the last section to derive a simple formula for the CP-asymmetry parameter $\epsilon_{1}$ in Eq. (6). The couplings give

$$
\left(\mathcal{N}^{\dagger} \mathcal{N}\right)_{1 j}=N_{3}^{2} \sum_{k} n_{k}^{2} U_{R 1 k}^{(N)} U_{R j k}^{(N) *}
$$

where the dominant contribution is given in this case by $k=3$,

$$
\left(\mathcal{N}^{\dagger} \mathcal{N}\right)_{1 j} \sim N_{3}^{2} U_{R 13}^{(N)} U_{R j 3}^{(N) *}
$$

As with the Majorana masses, third-generation dominance implies that simple expressions exist for 


$$
\left[\left(\mathcal{N}^{\dagger} \mathcal{N}\right)_{11},\left(\mathcal{N}^{\dagger} \mathcal{N}\right)_{12},\left(\mathcal{N}^{\dagger} \mathcal{N}\right)_{13}\right] \sim N_{3}^{2} s_{13}\left[s_{13}, s_{23}, 1\right] .
$$

The resulting CP-asymmetry parameter in Eq. (6) can now be expressed to leading order as

$$
\epsilon_{1} \sim \frac{3}{16 \pi} \frac{N_{3}^{2}}{v_{2}^{2}} \operatorname{Im}\left[\frac{\left(\mathcal{N}^{\dagger} \mathcal{N}\right)_{12}^{2}}{\left(\mathcal{N}^{\dagger} \mathcal{N}\right)_{11}} \frac{M_{1}}{M_{2}}+\frac{\left(\mathcal{N}^{\dagger} \mathcal{N}\right)_{13}^{2}}{\left(\mathcal{N}^{\dagger} \mathcal{N}\right)_{11}} \frac{M_{1}}{M_{3}}\right] .
$$

and one arrives at the simple result

$$
\epsilon_{1} \sim 10^{-1} s_{13}^{2} \sim 10^{-1} \frac{m_{u}}{m_{t}}
$$

where we have used Eqs. (30) and (35). We have also used $N_{3} \sim v_{2}$ since the largest Yukawa coupling in the neutrino Dirac mass matrix is similar $(\mathcal{N} \sim \mathcal{U})$ to the top quark Yukawa coupling which is close to one. One can understand that the contribution involving the mixing angle $s_{13}^{2}$ is the leading contribution in the following way: The dominant contribution to leptogenesis comes from the decay of the lightest Majorana neutrino $(i=1)$ and the dominant Yukawa couplings occur in the third generation $(j=3)$. One obtains an acceptable amount of baryon asymmetry if $\epsilon_{1} \sim 10^{-6}$; this indeed results if $s_{13} \sim \sqrt{m_{u} / m_{t}}$.

The dilution mass defined in Eq. (7) can be expressed as

$$
\tilde{m}_{1} \sim \frac{N_{3}^{2} s_{13}^{2}}{N_{3}^{2} \tilde{W}_{3} s_{13}^{2}}=\tilde{W}_{3}^{-1}
$$

using the third-generation-dominance that results from assumptions [A1] and [A2], Given the expression for $\tilde{W}_{3}$ in Eq. (31) it is clear that the dilution mass is related in all cases to the light neutrino masses. This is precisely the range of dilution mass that gives a large asymmetry as has been pointed out many times before as an attractive and natural feature of the leptogenesis scenario.

We now proceed to examine some special cases for the size of the dilution mass. Assumptions [A1] and [A2] allow us to identify the sizes of the mixing angles in the the mixing matrix $U_{L}^{(N)}$. For example $s_{23} \sim \sqrt{m_{c} / m_{t}}$. So Eq. (19) can be written as

$$
U_{L}^{(N)} \sim\left(\begin{array}{ccc}
1 & s_{12} & s_{13} \\
s_{12} & 1 & s_{23} \\
s_{13} & s_{23} & 1
\end{array}\right)
$$

Recall that the left-handed mixing angles are similar to the right-handed mixing angles according to our assumptions. Using Eq. (23) we have that

$$
\left(V_{L}^{\dagger} U_{L}^{(N)}\right)_{k 3}^{2} \sim\left(\begin{array}{c}
\max \left(s_{13}^{2}, \Theta_{12}^{2} s_{23}^{2}, \Theta_{23}^{2} \Theta_{12}^{2}, \Theta_{23}^{2} \Theta_{13}^{2} s_{23}^{2}, \Theta_{13}^{2}\right) \\
\max \left(s_{23}^{2}, \Theta_{23}^{2}\right) \\
1
\end{array}\right) .
$$

These elements together with Eqs. (31) and (38) allow one to determine the dilution mass. The quantities $s_{i j}$ are all small compared with one since they have been related to the (lefthanded) Cabibbo-Kobayashi-Maskawa (CKM) matrix elements, but the $\Theta_{i j}$ might or might 
not be small. From the CHOOZ data [5] we know that the mixing angle $\Theta_{13}$ must be small as long as there is no unnatural cancellation between this angle and the one involved in converting the weak basis to the mass basis for the charged leptons, c.f. Eq. (22). One can relate the dilution mass in Eq. (38) to the light neutrino masses using Eqs. (31), (32), and (40). The mass ratios between light neutrinos are

$$
\frac{m_{i}}{m_{j}} \sim \frac{\left(V_{L}^{\dagger} U_{L}^{(N)}\right)_{i 3}^{2}}{\left(V_{L}^{\dagger} U_{L}^{(N)}\right)_{j 3}^{2}} .
$$

One can investigate a number of cases. Without any fine-tuning one expects the angles $\Theta_{i j}$ to be of the same order as the angles $s_{i j}$. In that case, 1) one obtains

$$
m_{2} \sim \frac{m_{c}}{m_{t}} m_{3}, \quad m_{1} \sim \frac{m_{u}}{m_{t}} m_{3}
$$

from Eqs. (40) and (41). The dilution mass is $\tilde{m}_{1} \sim m_{3}$ from Eqs. (31) and (38). This does not give good agreement with the experimental data since $m_{2} \simeq \sqrt{\Delta m_{21}^{2}}$ is too small to reconcile it with the solar LMA data and atmospheric data neutrino data $m_{3} \simeq \sqrt{\Delta m_{32}^{2}}$. The neutrino masses inherit the large hierarchy from the up quark sector. The conclusion is that one needs some amount of fine-tuning to get masses in acceptable agreement with the solar LMA data.

2) If one accepts some fine-tuning so that the mixing angle $\Theta_{23}$ is large and order one rather than similar to $s_{23}$ and $\Theta_{12}$ remains small, then Eq. (40) reduces to

$$
\left(V_{L}^{\dagger} U_{L}^{(N)}\right)_{j 3}^{2} \sim\left(\begin{array}{c}
\Theta_{12}^{2} \\
1 \\
1
\end{array}\right) .
$$

Even in this case the determinant of the seesaw mass formula, Eq. (10), must satisfy

$$
m_{1} m_{2} m_{3}=m_{u} m_{c} m_{t}\left(\frac{m_{t}}{M_{3}}\right)^{3}
$$

Then since $\Theta_{23}$ is large one expects the mass eigenvalues to satisfy

$$
m_{2} \sim m_{3} \sim \sqrt{m_{c} m_{t}}\left(\frac{m_{t}}{M_{3}}\right)
$$

so that

$$
m_{1} \sim m_{u}\left(\frac{m_{t}}{M_{3}}\right)
$$

The masses can be consistent with the LMA solar and atmospheric neutrino data. Then the dilution mass is given by $\tilde{m}_{1} \sim m_{2} \sim m_{3}$ from Eqs. (31) and (38) and is in an acceptable range.

3) With additional fine-tuning both the mixing angles $\Theta_{12}$ and $\Theta_{23}$ can be made large. Eq. (40) reduces to 


$$
\left(V_{L}^{\dagger} U_{L}^{(N)}\right)_{j 3}^{2} \sim\left(\begin{array}{l}
1 \\
1 \\
1
\end{array}\right) .
$$

The light neutrino masses are all the same order so that from Eq. (44) one gets

$$
m_{1} \sim m_{2} \sim m_{3} \sim\left(m_{u} m_{c} m_{t}\right)^{1 / 3}\left(\frac{m_{t}}{M_{3}}\right),
$$

where $m_{i}$ cannot be larger than $m_{j}$ if $i<j$. Then the dilution mass is $\tilde{m}_{1} \sim m_{1}$. This solution does not offer any explanation for a hierarchy in neutrino masses.

In all three cases the dilution mass $\tilde{m}_{1}$ lies roughly in the range spanned by light neutrino masses

$$
m_{1} \lesssim \tilde{m}_{1} \lesssim m_{3} .
$$

It should be understood here that the $\lesssim$ means that $\tilde{m}_{1}$ could be outside the upper and lower ends of the range by an order one parameter.

More generally, and outside the assumptions of this paper, one can consider the possibility that the charged lepton mass matrix contributes to large mixing for both the solar neutrino and atmospheric netrino oscillations or for either one, though the charged lepton transformation matrix $U_{L}^{(E)}$ via Eq. (22).

\section{SUMMARY}

We have shown that based upon a limited number of reasonable assumptions about the neutrino sector motivated by grand unification, one obtains the universal expression in Eq. (37) for the dominant contribution to the CP-violation parameter $\epsilon_{1}$ that determines the amount of leptogenesis in the early universe. Furthermore the dilution mass $\tilde{m}_{1}$ is expressed in terms of mixing angles in the light neutrino masses and it naturally falls in the range needed to explain the baryon asymmetry of the universe. While these assumptions are not required to obtain the necessary lepton asymmetry to explain the observed baryon asymmetry of the universe, they provide enough constraints to allow one to relate the CPviolation in the heavy Majorana neutrino decays and the important Yukawa couplings of these heavy neutrinos to low-energy observables: fermion masses and mixing angles.

\section{ACKNOWLEDGMENTS}

This work was supported in part by the U.S. Department of Energy under Grant No. No. DE-FG02-91ER40661. 


\section{REFERENCES}

[1] S. Fukuda et al. [SuperKamiokande Collaboration], Phys. Rev. Lett. 86, 5651 (2001) hep-ex/0103032.

[2] S. Fukuda et al. [Super-Kamiokande Collaboration], Phys. Rev. Lett. 86, 5656 (2001) hep-ex/0103033.

[3] T. Toshito [SuperKamiokande Collaboration], hep-ex/0105023.

[4] [SNO Collaboration], nucl-ex/0106015.

[5] M. Apollonio et al. [CHOOZ Collaboration], Phys. Lett. B 466, 415 (1999) [hepex/9907037.

[6] M. C. Gonzalez-Garcia, Nucl. Phys. Proc. Suppl. 95, 100 (2001) [hep-ph/0010136].

[7] W. Buchmuller and M. Plumacher, Phys. Lett. B 511, 74 (2001) hep-ph/0104189.

[8] L. Covi, E. Roulet and F. Vissani, Phys. Lett. B 384, 169 (1996) hep-ph/9605319.

[9] W. Buchmuller and M. Plumacher, Phys. Lett. B 389, 73 (1996) hep-ph/9608308.

[10] M. Flanz, E. A. Paschos, U. Sarkar and J. Weiss, Phys. Lett. B 389, 693 (1996) [hepph/9607310.

[11] W. Buchmuller and M. Plumacher, Phys. Lett. B 431, 354 (1998) hep-ph/9710460.

[12] R. Rangarajan, U. Sarkar and R. Vaidya, Phys. Lett. B 442, 243 (1998) [hepph/9809304.

[13] A. Pilaftsis, Int. J. Mod. Phys. A 14, 1811 (1999) arXiv:hep-ph/9812256.

[14] M. S. Berger and B. Brahmachari, Phys. Rev. D 60, 073009 (1999) hep-ph/9903406].

[15] M. S. Berger, Phys. Rev. D 62, 013007 (2000) [hep-ph/9906490].

[16] M. Plumacher, hep-ph/9807557.

[17] G. C. Branco, T. Morozumi, B. M. Nobre and M. N. Rebelo, hep-ph/0107164.

[18] A. Pilaftsis, Phys. Rev. D 56, 5431 (1997) arXiv:hep-ph/9707235.

[19] M. Hirsch and S. F. King, hep-ph/0107014.

[20] D. Falcone and F. Tramontano, Phys. Rev. D 61, 113013 (2000) hep-ph/9910215.

[21] R. N. Mohapatra and X. Zhang, Phys. Rev. D 46, 5331 (1992).

[22] D. Falcone, Phys. Lett. B 475, 92 (2000) hep-ph/9912491.

[23] D. Falcone, Phys. Lett. B 479, 1 (2000) hep-ph/0002242].

[24] D. Falcone and F. Tramontano, Phys. Lett. B 506, 1 (2001) hep-ph/0101151.

[25] D. Falcone and F. Tramontano, Phys. Rev. D 63, 073007 (2001) hep-ph/0011053.

[26] F. Buccella, D. Falcone and F. Tramontano, hep-ph/0108172.

[27] M. Plumacher, Z. Phys. C 74, 549 (1997) hep-ph/9604229.

[28] M. Plumacher, Nucl. Phys. B 530, 207 (1998) hep-ph/9704231.

[29] S. Carlier, J. M. Frere and F. S. Ling, Phys. Rev. D 60, 096003 (1999) hep-ph/9903300.

[30] E. Ma, S. Sarkar and U. Sarkar, Phys. Lett. B 458, 73 (1999) hep-ph/9812276.

[31] H. Goldberg, Phys. Lett. B 474, 389 (2000) [hep-ph/9909477.

[32] K. Kang, S. K. Kang and U. Sarkar, Phys. Lett. B 486, 391 (2000) hep-ph/9906486.

[33] E. Nezri and J. Orloff, hep-ph/0004227.

[34] A. S. Joshipura and E. A. Paschos, hep-ph/9906498.

[35] A. S. Joshipura, E. A. Paschos and W. Rodejohann, JHEP 0108, 029 (2001) arXiv:hepph/0105175.

[36] W. Buchmuller and D. Wyler, arXiv:hep-ph/0108216.

[37] Z. Maki, M. Nakagawa and S. Sakata, Prog. Theor. Phys. 28, 870 (1962). 